\title{
A Narrative Review of Clinical researches of Acupuncture treatment for Depression using Neuroimaging method: Focusing on SCI papers
}

\author{
Dong Hyuk Lee ${ }^{1,2}$ \\ ${ }^{1}$ College of Korean Medicine, Sangji University \\ ${ }^{2}$ Research Institute of Korean Medicine, Sangji Univeristy
}

\begin{abstract}
Objectives: The purpose of this article was to investigate the current status of clinical studies of acupuncture treatment for depression using neuroimaging method, focusing on SCI papers.

Methods: We searched for clinical trial studies of acupuncture treatment for depression using neuroimaging method in the MEDLINE (Pubmed), OASIS, and RISS database. Once the online search was finished, studies were selected manually by the inclusion criteria. Finally, we analyzed the characteristics of selected articles and reviewed the neural substrates of acupuncture treatment in depression.

Results: Total eight studies were included in this study. The most frequently utilized modality was functional MRI. The most frequently selected acupoint for depression was GV20. Several studies revealed that acupuncture treatment could improve the symptoms of depression. In this manuscript, we demonstrated that neuroimaging techniques could capture the neural substrates associated with depression and acupuncture treatment may modulate the activation of brain areas which were impaired in depression in a different way from sham acupuncture.

Conclusions: Utilizing neuroimaging methods to explore neural mechanism of acupuncture treatment on depression would be helpful in clinical trials and more efforts should be needed in this fields.
\end{abstract}

$\overline{\text { Key Words }}$ : Depression, Acupuncture, Neuroimaging, fMRI, GV20

\section{Introduction}

Depression is a representative neuropsychiatric disease that causes symptoms not only in mood, but also in cognitive and motor functions. It also affects overall quality of life as well as social and occupational problems ${ }^{1)}$. According to the analysis from a cohort of more than 1 million people in Korea, the prevalence of depression in 2013 was $5.3 \%$, which nearly doubled compared to $2002^{2}$.
In this article, it was reported that the suicide rate was 3.8 times higher in depressed patients than in healthy subjects. These trends are thought to be worsened because of the pandemic of COVID-19. According to the 'COVID-19 National Mental Health survey' conducted in the $1^{\text {st }}$ quarter of 2021 in Korea, the overall mental health indicators, such as depression and suicidal impulse, worsened and the average score for depression increased more than twice compared to $2018^{3)}$.

- Received : 3 August 2021

- Revised : 17 September 2021

- Accepted : 5 November 2021

- Correspondence to : Dong Hyuk Lee

College of Korean Medicine, Sangji University, 83 Sangjidae-gil, Wonju-si, Gangwon-do 26339, Republic of Korea

E-mail: neurohani_ldh@sangji.ac.kr 
Treatment and management for depression can be largely divided into drug therapy and non-drug therapy. In case of drug therapy, selective serotonin reuptake inhibitors (SSRI), selective norepinephrine reuptake inhibitors (SNRI) are primarily considered and TCA (Tricyclic anti-depressant), other atypical antipsychotics, lithium, thyroid hormone can be utilized. Non-pharmacological therapies include several psycho-social treatments, such as interpersonal psychotherapy, cognitive behavioral therapy, mindfulness, cognitive awareness and problem-solving therapy ${ }^{4)}$.

For the treatment and management of depression, many researches are being actively performed in complementary and alternative medicines including traditional Korean medicine. Among them, acupuncture treatment has been applied for a variety of neurological and psychiatric disorders, such as cerebrovascular disease, Parkinson's disease, dementia and mental illness. However, clear mechanisms of acupuncture in clinical practice are still uncertain and neuroimaging methods are being utilized as one of the non-invasive techniques to identify them. Neuroimaging is a generic term for various techniques for imaging the structure and function of the brain. Magnetic Resonance Imaging (MRI), Diffusion Tensor Imaging (DTI) are those of structural imaging techniques, and functional MRI (fMRI), Perfusion MRI, Positron Emission Tomography (PET), and Single Photon Emission Computed Tomography (SPECT) are applied as functional neuroimaging.

In order to investigate the effect of traditional Korean medicine or acupuncture treatment for depression using neuroimaging, it would be necessary to explore and review the current status of the studies so far. Therefore, in this article, we searched and reviewed the clinical papers of acupuncture treatment for depression applying neuroimaging that have been published in Science Citation Index (SCI) so far, as a basic reference for future acupuncture-related translational studies.

\section{Methods}

\section{Literatures subject to the research}

A systematic search was conducted using OASIS(https://oasis.kiom.re.kr), RISS(http://www. riss.kr/index.do) and PubMed(https://www.ncbi. nlm.nih.gov/pubmed) for literature search on clinical studies of acupuncture for depression using neuroimaging. OASIS is a typical Korean medicine database provided by Korea Institute of Oriental Medicine(KIOM). RISS is an open public service allowing academic resources by Korea Education \& Research Information Service (KERIS). PubMed is a representative bibliographic database provided by the National Center for Biotechnology Information (NCBI). By 14 July 2021, the search included all the literature provided in the database, and the MeSH term was composed of a combination of 'depression', 'acupuncture', and 'neuroimaging' as keywords. In addition, the term of 'MRI, 'DTI', 'PET', or 'SPECT' were used as keywords instead of the term 'neuroimaging' to search more closely for studies associated with respective neuroimaging method.

\section{Research methods}

The author finally selected the articles to be evaluated according to the following criteria from 
the first searched papers. In addition, the author excluded papers according to the exclusion criteria. The selection criteria were as follows. 1) A study on acupuncture for depression (published journal not thesis) 2) A clinical research 3) A study using neuroimaging techniques (Table 1). Meanwhile, the exclusion criteria were as follows. 1) A study not related to depression, acupuncture, or neuroimaging methods 2) Duplicate paper 3) Unavailable original text 4) Review paper or protocol study 5) Non-clinical research.

Next, the following characteristics were analyzed, along with the general characteristics of the selected studies (author, publication journal, publication year).

1) Modality of neuroimaging methods

2) Intervention: type of acupuncture and control group

3) Acupoints

4) Study design: sample size, type of subjects, RCT

5) Clinical measurements

6) Neuroimaging index in the analysis

\section{Results}

\section{The Selection of research papers}

For the first time, 43 papers were searched for 'depression', 'acupuncture', and 'neuroimaging'.
When MRI, PET, SPECT, and DTI were searched instead of neuroimaging, 83, 8, 1, and 3 papers were searched, respectively. After manually checking whether the conditions are met, duplicate papers, animal experiments, papers unrelated to depression, unavailable original text, papers unrelated to acupuncture treatment, papers unrelated to neuroimaging, and review or protocol papers were excluded. Total 8 papers were selected as target articles (Fig. 1).

\section{General characteristics of selected articles}

Publication year, published journal name, utilized neuroimaging technique, treatment method, RCT status (control group), and type and numbers of subjects in each article were summarized (Table 2). All eight papers, except for two, were studies conducted in China, and the applied imaging techniques were MRI in one and fMRI in the rest. The articles have been published in various journals in the field of neuroscience or psychiatry, such as 'Neuroimage: Clinical', 'Frontiers in Human Neuroscience', 'Journal of Psychiatric Research', and 'Brain Imaging and Behavior'.

\section{The frequency analysis of acupoints}

The Acupoints used in 8 clinical studies have been summarized (Table 3). Baihui(GV20) was the most commonly used acupoint, which was

Table 1. Types of Neuroimaging modality

\begin{tabular}{lll}
\hline & \multicolumn{1}{c}{ Structural } & \multicolumn{1}{c}{ Functional } \\
\hline \multirow{3}{*}{ Modality } & Magnetic Resonance Imaging (MRI), Diffusion & Functional MRI (fMRI), Perfusion MRI, Positron \\
& Tensor Imaging (DTI) & Emission Tomography (PET), Single Photon Emission \\
& & Computed Tomography (SPECT) \\
\hline
\end{tabular}




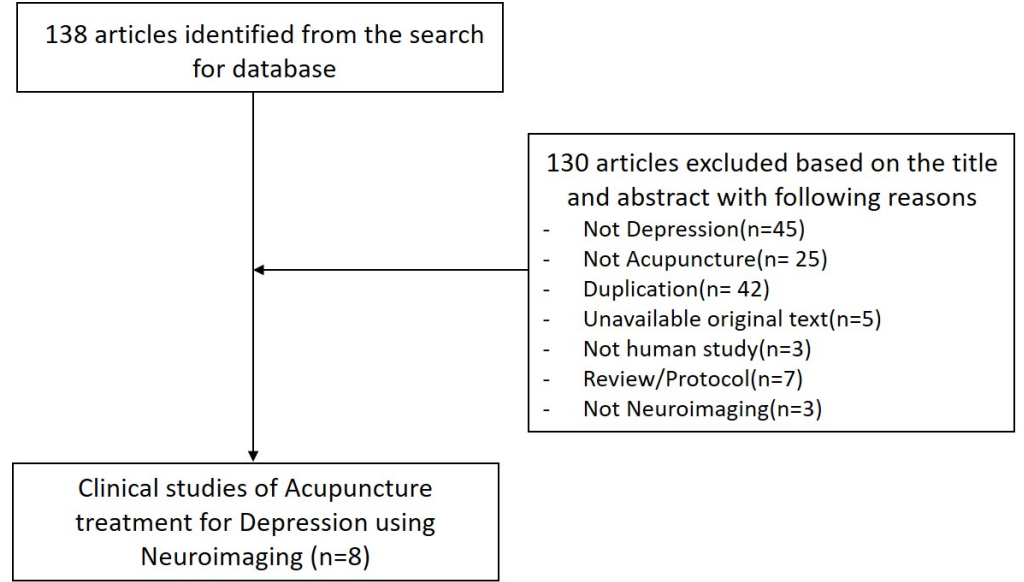

Fig. 1. Flow chart of the study selection process

applied in 4 out of 8 papers ${ }^{5,8,11,12)}$. In three articles $^{8,11,12)}$, GV20 was applied as a single acupoint for electroacupuncture. In the remaining paper $^{5}$, it was used in combination with Yintang
(EX-HN3) for electroacupuncture. In this study, individual acupoints were also used according to the subject's symptoms (ex. headache, dizziness, insomnia, digestive symptoms, pain). Moreover,

Table 2. Characteristics of the Studies using Neuroimaging

\begin{tabular}{|c|c|c|c|c|c|}
\hline Author (Year) & Journal & Modality & Intervention & $\begin{array}{c}\mathrm{RCT} \\
\text { (Control) }\end{array}$ & Subjects \\
\hline Duan $(2011)^{5}$ & $\begin{array}{l}\text { Chin Journal of Integrative } \\
\text { Medicine }\end{array}$ & MRI, H-MRS & $\begin{array}{l}\text { Electro-Acu+ } \\
\text { Fluoxetine }\end{array}$ & O (Fluoxetine) & $\begin{array}{r}75 \text { subjects } \\
\text { (depression) }\end{array}$ \\
\hline Quah-Smith $(2012)^{6}$ & Medical Acupuncture & fMRI & laser Acu & $\mathrm{X}$ & $10 \mathrm{MD}$ \\
\hline Quah-Smith (2013) ${ }^{7}$ & Medical Acupuncture & fMRI & laser Acu & $\mathrm{X}$ & $\begin{array}{l}10 \mathrm{MD}, \\
10 \mathrm{HC}\end{array}$ \\
\hline Deng $(2016)^{8}$ & $\begin{array}{l}\text { Frontiers in Human } \\
\text { Neuroscience }\end{array}$ & fMRI & Electro-Acu & $\mathrm{X}$ & $\begin{array}{l}29 \mathrm{MD}, \\
29 \mathrm{HC}\end{array}$ \\
\hline Wang $(2016)^{9}$ & Neuroimage: Clinical & fMRI & $\begin{array}{l}\text { Acu+ } \\
\text { Fluoxetine }\end{array}$ & $\begin{array}{l}\mathrm{O}(\text { Sham }+ \\
\text { Fluoxetine })\end{array}$ & $46 \mathrm{MD}$ \\
\hline Wang $(2017)^{10}$ & $\begin{array}{l}\text { Journal of Psychiatric } \\
\text { Research }\end{array}$ & fMRI & $\begin{array}{c}\text { Acu+ } \\
\text { Fluoxetine }\end{array}$ & $\begin{array}{c}\mathrm{O}(\text { Sham }+ \\
\text { Fluoxetine) }\end{array}$ & $46 \mathrm{MD}$ \\
\hline Duan $(2020)^{11}$ & Brain Imaging and Behavior & fMRI & Electro-Acu & $\mathrm{X}$ & $\begin{array}{l}30 \mathrm{MD} \\
29 \mathrm{HC}\end{array}$ \\
\hline Wei $(2021)^{12}$ & $\begin{array}{l}\text { Neuropsychiatric Disease and } \\
\text { Treatment }\end{array}$ & fMRI & Electro-Acu & $\mathrm{X}$ & $\begin{array}{l}20 \text { subjects } \\
\text { (depression) }\end{array}$ \\
\hline
\end{tabular}

MRI: Magnetic Resonance Imaging

H-MRS: Proton Magnetic Resonance Spectroscopy

fMRI: functional Magnetic Resonance Imaging

Acu: Acupuncture

Electro-Acu: Electroacupuncture

MD: Major depressive disorder patients 
in two studies ${ }^{9,10)}$, only abdominal acupoints (Zhongwan (RN12), Xiawan (RN10), Qihai (RN6), Guanyuan (RN4), Shangqu (KL17), Huaroumen (ST24), Qipang (Extra)) were utilized. Finally, there were two consecutive studies ${ }^{6,7)}$ that combined the acupoints of Qimen (LR14), Ququan (LR8), Juque (CV14), Shenmen (HT7) for laser acupuncture.

\section{The analysis of study design and control group}

Among the studies, RCT studies were three ${ }^{5,9,10)}$ and the others were case series studies ${ }^{6,7,8,11,12)}$. There were two studies ${ }^{9,10)}$ using sham acupuncture. The sham acupuncture was lightly tapped with a plastic tube without needle insertion at the same location of verum acupuncture. The control group in the rest of the RCT study was the single administration of Fluoxetine, one of the SSRIs. The treatment group included electroacupuncture ${ }^{8,11,12)}$, laser acupuncture ${ }^{6,7)}$, and the combination of acupuncture/electroacupuncture and Fluoxetine ${ }^{5,9,10)}$.

Electroacupuncture was performed at $1 \mathrm{~Hz}^{8,11)}$ or $2 \mathrm{~Hz}^{5,12)}$ and laser acupuncture was conducted by stimulating each acupoint 4 times for 20 seconds with an intensity of $25 \mathrm{~mW}^{6,7)}$. The depths of the needle were represented as not mentioned ${ }^{5,6,7)}$, 1-1.5 $\mathrm{cm}^{8,11)}, 1.5-2 \mathrm{~cm}^{9,10,12)}$. There were 4 studies $^{5,8,11,12)}$ that confirmed the deqi sensation from acupuncture. In these studies, the frequency and intensity of sensation were measured with a self-rating (VAS) of 100 points $^{8,11)}$, or 10 points $^{12}$.

The study design including duration or frequency of the intervention can be divided according to the purpose of the study. The aims were to achieve an immediate response after acupuncture stimulation $^{6,78,11,12)}$ or to confirm the long-term effects of acupuncture treatment ${ }^{5,9,10)}$ (Table 4). In case of $5^{6,7,8,11,12)}$ out of 8 , acupuncture stimulation was one-time and the time was about $15-20$ minutes in order to measure the immediate effect of acupuncture stimulation. On the other hand, in the study for long-term effects of acupuncture, it was administered for 6-8 weeks of duration and at intervals of once a day ${ }^{5)}$ or 1 time/3 days ${ }^{9,10)}$. And the clinical improvements and change of neuroimaging indicators were investigated between the baseline and the completion.

\section{Clinical indicators, neuroimaging index analysis and results of acupuncture}

Table 3. Acupoints of the Studies using Neuroimaging

\begin{tabular}{|c|c|c|}
\hline Author (Year) & Acupoints & Deqi \\
\hline Duan $(2011)^{5}$ & Baihui (GV20), Yintang (EX-HN3) + additional acupoints according to individual symptoms & $\mathrm{O}$ \\
\hline Quah-Smith $(2012)^{6}$ & Qimen (LR14), Ququan (LR8), Shenmen (HT7), Juque (CV14), Taixi (KI3) & $\mathrm{X}$ \\
\hline Quah-Smith $(2013)^{7}$ & Qimen (LR14), Ququan (LR8), Juque (CV14), Shenmen (HT7) & $\mathrm{X}$ \\
\hline Deng $(2016)^{8}$ & Baihui (GV20) & $\mathrm{O}$ \\
\hline Wang $(2016)^{9}$ & $\begin{array}{l}\text { Zhongwan (RN12), Xiawan (RN10), Qihai (RN6), Guanyuan (RN4), Shangqu (KL17), } \\
\text { Huaroumen (ST24), Qipang (Extra) }\end{array}$ & $\mathrm{X}$ \\
\hline Wang $(2017)^{10}$ & $\begin{array}{l}\text { Zhongwan (RN12), Xiawan (RN10), Qihai (RN6), Guanyuan (RN4), Shangqu (KL17), } \\
\text { Huaroumen (ST24), Qipang (Extra) }\end{array}$ & $\mathrm{X}$ \\
\hline Duan $(2020)^{11}$ & Baihui (GV20) & $\mathrm{O}$ \\
\hline Wei $(2021)^{12}$ & Baihui (GV20) & $\mathrm{O}$ \\
\hline
\end{tabular}




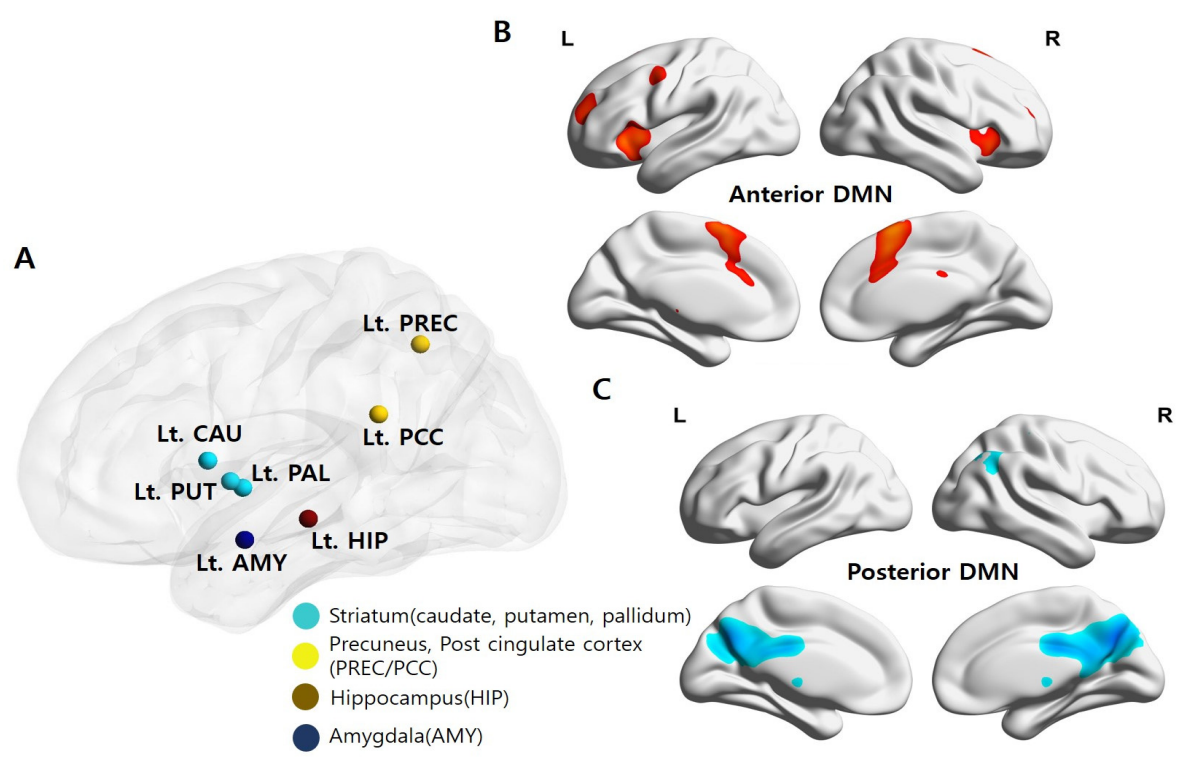

Fig. 2. Target Regions of Interest (ROIs) used in the studies included in this review. A: dorsal/ventral striatum, PC/PCC ROls, Hippocampus, Amygdala B: representative of anterior DMN area (from ICA) C: representative of posterior DMN area (from ICA)

The clinical index, results of neuroimaging measurements and clinical improvements from acupuncture treatment were simply summarized (Table 4, 5). Moreover, for better understanding, the target ROIs utilized in the studies included in this review are visually presented (Figure 2). First of all, Duan (2011) ${ }^{5}$ ) used MRI and Proton magnetic resistance spectroscopy (H-MRS) to measure the volume and metabolite of hippocampus and showed that these metabolites changed in the electroacupuncture group. Metabolic changes were significantly associated with pre-treatment depression scores (17-item Hamilton Scale for Depression). In addition, significant changes in depression scores appeared in the electroacupuncture group, and it was a study that confirmed that there was a significant correlation between these therapeutic effects and changes in metabolites. However, there was no significant difference in the volume of the hippocampus between the treatment group and the control group. Quah-Smith (2012) ${ }^{6}$ ) used laser acupuncture to investigate its biological plausibility as an antidepressant treatment. Five different acupoints related to Liver, Heart, conception vessel and Kidney meridian were evaluated. Regions with significantly increased activation and deactivation included fronto-limbic -striatal regions and cerebellum, parts of Default mode network (DMN).

As a follow-up study, Quah-Smith (2013) $)^{7)}$ applied laser acupuncture to explore how laser acupuncture works differently in the brain of depressed patients and normal groups. It is known that the brain has a resting state large scale network called the DMN. In this study, DMN was extracted by Independent Component Analysis. 
It was confirmed that laser acupunctures induced changes in the frontal areas of DMN in the normal group, while in the depression group, they induced changes in the lower parietal lobes or cerebellum. When stimulating the acupoints (Qimen (LR14), Ququan (LR8), Juque (CV14)), it was possible to control the anterior DMN in the normal group and the posterior DMN in the patient group, and there was no difference between groups in the case of Shenmen (HT7), unlike their previous study6). Deng $(2016)^{8)}$ also wanted to clarify the relationship between DMN changes and depression. They performed a functional connectivity (FC) analysis by setting the precuneus, and posterior cingulated gyrus (PC/PCC), which were the components of DMN, as seeds. In the depression group, the connectivity with other areas of DMN including middle prefrontal cortex and ACC was altered and the stimulation of electroacupuncture on GV20 modulated these alterations.

Wang $(2016)^{9)}$ examined whether the real acupuncture using abdominal acupoints and fluoxetine showed significant differences in the improvement of depression compared to the sham acupuncture and fluoxetine, and in the functional

Table 4. Features of intervention and clinical measurements on the studies using Neuroimaging

\begin{tabular}{|c|c|c|c|}
\hline Author (Year) & $\begin{array}{c}\text { Number of Intervention or } \\
\text { Duration }\end{array}$ & Clinical index & long-term changes in clinical index \\
\hline Duan $(2011)^{5}$ & $\begin{array}{l}\text { once a day }(30 \mathrm{~min}), 6 \text { times a } \\
\text { week ( } 6 \text { weeks })\end{array}$ & 17-item HAMD & $\begin{array}{l}\text { The reduction rate of HAMD was significantly higher } \\
\text { in EA group, compared to control group (reducing } \\
\text { rate: } 56.8(15.2) \text { (EA) vs 50.8(17.6) (Fluoxetine), } \\
\mathrm{p}=0.043 \text { ) }\end{array}$ \\
\hline Quah-Smith $(2012)^{6}$ & $\begin{array}{l}\text { Immediate response during } \\
\text { laser acupuncture }\end{array}$ & $\begin{array}{l}\text { BDI, MADRS, } \\
\text { HAMD }\end{array}$ & NA (not measured after the intervention) \\
\hline Quah-Smith $(2013)^{7}$ & $\begin{array}{l}\text { Immediate response during } \\
\text { laser acupuncture }\end{array}$ & $\begin{array}{l}\text { BDI, MADRS, } \\
\text { HAMD }\end{array}$ & NA (not measured after the intervention) \\
\hline $\operatorname{Deng}(2016)^{8}$ & $\begin{array}{l}\text { Immediate response after } \\
\text { electro-acupuncture }\end{array}$ & $\begin{array}{l}\text { SDS, SAS, } \\
\text { 17-HDRS }\end{array}$ & NA (not measured after the intervention) \\
\hline Wang $(2016)^{9}$ & $\begin{array}{l}\text { once a day for the first three } \\
\text { days, and subsequently once } \\
\text { every } 3 \text { days for the reminder } \\
\text { (20min, } 8 \text { weeks) }\end{array}$ & MADRS, SDS & $\begin{array}{l}\text { The verum acupuncture group showed significantly } \\
\text { greater clinical improvement (post - pre-treatment, } \\
\text { MADRS, } \mathrm{F}=10.86, \mathrm{p}<0.01 ; \mathrm{SDS}, \mathrm{F}=8.21, \mathrm{p}<0.01 \text { ), } \\
\text { compared to sham group }\end{array}$ \\
\hline Wang $(2017)^{10}$ & $\begin{array}{l}\text { once a day for the first three } \\
\text { days, and subsequently once } \\
\text { every } 3 \text { days for the reminder } \\
\text { (20min, } 8 \text { weeks) }\end{array}$ & MADRS, SDS & $\begin{array}{l}\text { The verum acupuncture group showed significantly } \\
\text { greater clinical improvement } \\
\text { (post - pre-treatment (verum vs sham), MADRS, } \Delta \text { : } \\
-17.5(7.9) \text { vs }-8.8(7.9), \mathrm{p}<0.01 \text {; SDS, } \Delta:-21.0(9.1) \text { vs } \\
-12.5(8.7), \mathrm{p}<0.01 \text { ), compared to sham group }\end{array}$ \\
\hline Duan $(2020)^{11}$ & $\begin{array}{l}\text { Immediate response after } \\
\text { electro-acupuncture }\end{array}$ & $\begin{array}{l}\text { SDS, SAS, } \\
17 \text {-HDRS }\end{array}$ & NA (not measured after the intervention) \\
\hline Wei $(2021)^{12}$ & $\begin{array}{l}\text { Immediate response after } \\
\text { electro-acupuncture }\end{array}$ & HRSD-17 & NA (not measured after the intervention) \\
\hline
\end{tabular}

HAMD/HDRS/HRSD: Hamilton Scale for Depression

BDI: Beck Depression Inventory

MADRS: Montgomery and Asberg Depression Rating scale

SDS: Self rating depression scale

SAS: Self rating anxiety scale 
Table 5. Analysis Index and results of the studies using Neuroimaging

\begin{tabular}{|c|c|c|}
\hline $\begin{array}{l}\text { Author } \\
\text { (Year) }\end{array}$ & $\begin{array}{l}\text { Methods in } \\
\text { Neuroimaging }\end{array}$ & Neuroimaging results of Acupuncture treatment \\
\hline Duan $(2011)^{5}$ & $\begin{array}{l}\text { Hippocampal volume, } \\
\text { ratio of several } \\
\text { metabolites from } \\
\text { hippocampus }\end{array}$ & $\begin{array}{l}\text { There was a negative correlation between NAA/Cr change ratio and the HAMD } \\
\text { scores before treatment }(\mathrm{p}=0.006(\mathrm{Rt}), 0.0089(\mathrm{Lt})) \text { in the EA group. There was } \\
\text { positive relevance between the hippocampal NAA/Cr change ratio and the } \\
\text { therapeutic effect (r: } 0.22(\mathrm{Rt}, \mathrm{p}=0.04), 0.24(\mathrm{Lt}, \mathrm{p}=0.03) \text { ), and between hippocampal } \\
\text { Cho/Cr change ratio and the therapeutic effect (r: } 0.50(\mathrm{Rt}, \mathrm{p}=0.04), 0.53(\mathrm{Lt} \text {, } \\
\mathrm{p}=0.03) \text { ), only in the EA group }\end{array}$ \\
\hline $\begin{array}{l}\text { Quah-Smith } \\
(2012)^{6}\end{array}$ & Brain activation & $\begin{array}{l}\text { Stimulation of the acupoints LR } 8 \text {, LR } 14 \text { and CV } 14 \text { altered activity in many brain } \\
\text { regions relevant to mood (lingual gyrus, cuneus, cerebellum, inferior parietal area), } \\
\text { whereas HT } 7 \text { produced activation of middle frontal gyrus and deactivation of } \\
\text { middle temporal gyrus only. The stimulation of KI } 3 \text { did not have any significant } \\
\text { brain effects. In this study, the modulation of DMN by low-intensity laser } \\
\text { acupuncture was also revealed. }\end{array}$ \\
\hline $\begin{array}{l}\text { Quah-Smith } \\
(2013)^{7}\end{array}$ & $\begin{array}{l}\text { Default mode network } \\
\text { (DMN) pattern (by } \\
\text { Independent component } \\
\text { analysis) }\end{array}$ & $\begin{array}{l}\text { The healthy subjects had significant modulation of DMN in the frontal region at the } \\
\text { medial frontal gyrus for three acupoints. For depressed participants, the DMN } \\
\text { modulation occurred at the inferior parietal cortex and the cerebellum. Laser } \\
\text { acupuncture on LR8, LR14 and CV14 tended to modulate anterior DMN in healthy } \\
\text { subjects, but affect posterior DMN in depressed subjects. }\end{array}$ \\
\hline Deng $(2016)^{8}$ & $\begin{array}{l}\text { Resting state seed-based } \\
\text { functional connectivity } \\
\text { (FC) of PC/PCC (seed as } \\
\text { a DMN) }\end{array}$ & $\begin{array}{l}\text { Compared to healthy subjects, patients had abnormal patterns of the DMN including } \\
\text { the right middle prefrontal cortex, bilateral angular gyrus and ACC with the } \\
\text { PC/PCC. GV20-related EA stimulation may modulate the DMN in patients with } \\
\text { first-episode, drug-naive MDD, related to the left middle prefrontal cortex, left } \\
\text { angular gyrus, bilateral hippocampus and ACC. }\end{array}$ \\
\hline Wang $(2016)^{9}$ & $\begin{array}{l}\text { Resting state seed-based } \\
\text { FC of amygdala }\end{array}$ & $\begin{array}{l}\text { The verum acupuncture group showed significantly increased rsFC of the amygdala } \\
\text { with brain regions associated with emotion and affect modulation (sgACC, pgACC, } \\
\text { parahippocampus, putamen). } \\
\text { The FC of amygdala were also significantly associated with a reduction in symptom } \\
\text { severity (MADRS, SDS) (p=0.02, } 0.01 \text {, respectively). }\end{array}$ \\
\hline Wang $(2017)^{10}$ & $\begin{array}{l}\text { Resting state seed-based } \\
\text { FC of dorsal/ventral } \\
\text { striatum }\end{array}$ & $\begin{array}{l}\text { After } 8 \text { weeks, compared with sham group, the verum acupuncture group showed } \\
\text { significantly increased rsFC of the corticostriatal reward circuits (iVS-medial } \\
\text { prefrontal cortex(rMPFC), vrP- amygdala/parahippocampus(AMY/PHC), } \\
\text { dC-middle temporal gyrus) and decreased striatal-cerebellar } \\
\text { regions(vrP-dorsolateral prefrontal cortex(DLPFC), dC-crerebellar tonsil). The } \\
\text { rsFC changes in the cortico-striatum are also significantly correlated with a } \\
\text { reduction in symptom severity (vrP-AMY/PHC:p=0.048(MADRS), } 0.048 \text { (SDS); } \\
\text { dC-MTG: } \mathrm{p}=0.009 \text { (MADRS), } 0.016(\mathrm{SDS}) \text { ). }\end{array}$ \\
\hline Duan $(2020)^{11}$ & $\begin{array}{l}\text { Resting state seed-based } \\
\text { FC of amygdala }\end{array}$ & $\begin{array}{l}\text { The results validated the research hypothesis that compared to healthy subjects, there } \\
\text { exists aberrant AN (amygdala network) in patients with MDD, mainly increased } \\
\text { amygdala FC with hippocampus, precuneus, precentral gyrus and angular gyrus and } \\
\text { decreased amygdala FC with OFC (orbital frontal gyrus). Secondly, amelioration of } \\
\text { aberrant AN in MDD patients was induced by EAS at GV20. GV20 could affect the } \\
\text { FC of the amygdala, mainly increased FC in amygdala with DLPFC, and decreased } \\
\text { FC in amygdala with periaqueductal gray matter (PAG) and insula. }\end{array}$ \\
\hline Wei $(2021)^{12}$ & $\begin{array}{l}\text { Regional Homogeneity } \\
\text { (ReHo) }\end{array}$ & $\begin{array}{l}\text { EA at GV20 could first instantly activate bilateral postcentral gyri, right calcarine } \\
\text { gyrus, right cuneus. Pairwise comparison between sustained effect (Phase } 3 \text { ) and } \\
\text { instant effect (Phase } 2 \text { ) in EA stimulation found significantly decreased ReHo values } \\
\text { in the posterior cingulate cortex (PCC), right angular gyrus, precuneus (Part of } \\
\text { DMN). The positive correlations between the HRSD-17 scores and the ReHo values } \\
\text { of right angular gyrus }(r=0.45(\mathrm{p}=0.048) \text {, before acupuncture, Phase } 1) \text {, right and left } \\
\text { postcentral gyrus (Rt: } r=0.68(\mathrm{p}=0.001), \mathrm{Lt}: \mathrm{r}=0.62(\mathrm{p}=0.004) \text {, after acupuncture, } \\
\text { Phase } 3 \text { ) were found. }\end{array}$ \\
\hline
\end{tabular}


connectivity centered on amygdala, which is well known for regions associated with emotion. As a result, in the combined treatment group, the connectivity between several brain regions associated with emotion and amygdala increased and this change was significantly correlated with the clinical improvements. Wang $(2017)^{10)}$ had the similar concept as the previous study ${ }^{9}$, but the connectivity analysis was performed by placing the region of interest (ROI) in the corticostriatal reward circuit, holding the dorsal and ventral striatum as a seed. As a result, the genuine acupuncture and the fluoxetine group increased the connectivity of the reward circuit between cortex and striatum compared to the sham group and decreased the connectivity between striatum-cerebellum. In addition, changes in the connectivity between the cerebral cortex and the striatum showed significant association with clinical improvements in depression.

Duan $(2020)^{11)}$ observed connectivity changes centered on amygdala, similar with Wang $(2016)^{9)}$, but had a difference in the selection of acupoints and the study design. They sought to examine an immediate response to acupuncture, not a long-term effect of acupuncture. In this study, the connectivity between amygdala and certain areas (hippocampus, precuneus, and precentral gyrus) in depressed patients increased, while the connectivity with the orbital frontal gyrus was diminished. Also, the stimulation of electroacupuncture on GV20 could modulate these connectivity alterations. Wei (2021) calculated the value of Regional homogeneity (ReHo), one of the methods of measuring brain intrinsic activity using BOLD signals, to examine the changes before, during and after the electroacupuncture stimulation on GV20. As an immediate effect, the acupuncture stimulation activated signals of both postcentral gyri, calcarine gyrus, and cuneus, and reduced signals from DMN regions such as PCC, angular gyrus, and precuneus as a delayed effect after the stimulation. The ReHo values of angular gyrus and postcentral gyrus showed positive correlation with depression scores before and after acupuncture, respectively.

\section{Discussion}

In the previous report on May 21, the OECD announced that the global mental health problem triggered by COVID-19 were causing great economic losses. Here, the OECD compared the prevalence of depression among several countries between Pre-COVID and 2020. The results showed that the number of people suffering from depression has more than doubled in most countries after the pandemic of COVID-19. In case of Korea, the situation is more serious, with a prevalence of about 4 out of $10(38.6 \%)^{13)}$.

The patients with depression tend to complain of not only mood disorders but also various physical symptoms including chronic pain, headache, joint pain, stuffiness, abdominal discomfort, chills, cold sweat, burns and thirst ${ }^{14)}$. In the clinical practices of traditional Korean medicine, most depressive patients come to the hospital while taking antidepressants. In our opinion, there would be some demand for traditional Korean medicine to make up for the lack of therapeutic effect in conventional medicine, deal with various side 
effects or systemic symptoms as described above while taking antidepressants.

Many efforts to conquer depression have been made on complementary and alternative medicines as well as conventional medicine. Acupuncture treatment has been utilized in numerous neuropsychiatric diseases and its safety has been proved for a long time. Therefore, acupuncture is thought to have a potential role in the treatment of depression. Recently, the traditional Korean medicine clinical practice guideline for depression is developed ${ }^{15}$, but the systematic studies on the mechanism of acupuncture in depression are still relatively lacking. In this situation, the latest neuroimaging technique can be a useful tool to elucidate the mechanism of acupuncture in depression. Therefore, we investigated and reviewed the current status of clinical studies of acupuncture treatment with neuroimaging methods related to depression.

Neuroimaging refers to a technique for imaging the brain at the structural, functional and molecular level in various ways. Structural images include MRI using the principle of nuclear magnetic resonance, and DTI to track white matter bundles using the diffusion properties of water molecules. Functional images contain fMRI to measure neuronal activity, SPECT or perfusion images to check the degree of cerebral blood flow, FDG-PET to reflect intracerebral glucose metabolism, and amyloid or tau PET to measure the protein aggregation in neurodegenerative brain diseases. Neuroimaging techniques are being vigorously studied as biomarkers that reveal diagnosis or prognosis of the various disease such as Alzheimer's disease $^{16)}$, depression ${ }^{17)}$, schizophrenia ${ }^{18)}$,
Parkinson's disease ${ }^{19)}$.

Through the total 8 articles, Baihui (GV20) was the most commonly used acupoint. Actually, Baihui (GV20), which was a point on the Governor Vessel and located on the vertex of the head, was one of the most famous acupoints in traditional Korean medicine. It is also the acupoint where the qi(energy) of the whole body gathers. According to the principles of traditional Korean medicine and its location, it is a common acupoint utilized in neurological and psychiatric diseases, including dizziness, headache, anxiety, cognitive impairment, $\mathrm{MDD}^{20)}$.

There are two studies applying abdominal acupoints, which are Zhongwan (RN12), Xiawan (RN10), Qihai (RN6), Guanyuan (RN4), Shangqu (KL17), Huaroumen (ST24), Qipang (Extra), in particular. These acupoints is based on the principles of traditional Korean medicine that Shenque (CV8) plays a central role in promoting and regulating the flow of qi. They are also close to important meridians and can easily communicate with internal organs through Governor Vessel and Conceptional Vessel. There is a preliminary study showing that these points are effective for depression $^{21)}$. In addition, there have been two studies of laser acupuncture using acupoints including Qimen (LR14), Ququan (LR8), Juque (CV14), Shenmen (HT7) and Taixi (KI3) Ququan (LR8) is useful for turning off 'Liver fire', as a 'water point' on the Liver meridian. Qimen (LR14), Juque (CV14) are the 'alarm points' of the Liver meridian and Heart meridian. Although the acupoint of Shenmen (HT7), which is the origin point of the Heart meridian, is one of the most common acupoints for insomnia or 
anxiety, it did not specifically work to show the difference between depression and control group. The laser stimulation on Taixi (KI3) did not show significant activation or deactivation in depression group. In the future, it is thought that studies using the combination of Saam acupuncture, body acupuncture or scalp acupuncture could be possible as well.

The neuroimaging studies using acupuncture for depression were largely researches that explored the immediate response after acupuncture stimulation or long-term effects of acupuncture after treatment. Through this article, it is confirmed that functional images were utilized more than structural images. Since depression is not a neurodegenerative disease or geriatric disorders inducing cortical atrophy or volumetric loss, it is thought that functional images exploring activation or connectivity patterns in the brain were preferred. Also, in case of depression, research on imaging biomarker is relatively lacking compared to neurodegenerative disease. However, if further studies about the effects of acupuncture on depression are conducted in the fields of neuroimaging, it is necessary to utilize various modalities such as perfusion imaging, SPECT and FDG-PET.

In the analyses of neuroimaging index obtained from the BOLD signal (ReHo, ICA map, seed -based FC) in fMRI, it was confirmed that the depression group showed different activation pattern compared to normal group. The region of interest (ROIs) was amygdala, which is closely related to emotion, hippocampus, which is one of the main areas affected by stress, and striatum, which is associated with reward circuit known to be abnormal in depression. Moreover, the alteration of Default mode network (DMN) related to memory, self-referential processing and decision making was explored. There were many pattern differences in the area of frontal, temporal lobe, insula, anterior cingulate cortex, precuneus, DMN, and precentral gyrus, which are related to cognition, self-reference, sensation. In the study on the long-term effects of acupuncture, the difference of activation pattern between real acupuncture and sham acupuncture was investigated. In case of depression, the DMN region tends to be hyperactivated, and this activation was shown to be regulated through acupuncture treatment. Furthermore, the group of verum acupuncture showed more significant clinical improvements compared to sham group. Finally, by investigating the brain regions showing a significant correlation with clinical improvement, the underlying neural substrates of genuine acupuncture in modulating brain activation were explored.

There are some cautions to apply neuroimaging in the study. In general, since the correlation between certain indicators of brain regions and clinical information can only be obtained, experimental research should be accompanied to confirm the direct causality. Also, the preprocessing steps to improve signal to noise ratio (SNR) and the methods to deal with multiple comparison problem are very crucial. In this respect, some of the published articles were relatively liberal in the degree of statistical significance.

There are several limitations in this article. First, this study did not include various databases other than PubMed, OASIS and RISS. Secondly, 
qualitative evaluation used in many SR studies was not performed. Therefore, as the title suggests, this study should be viewed as a narrative review. It is considered that a systemic literature review would be needed in the future.

For a more systematic study in the future, first, it is necessary to promote clinical researches using various acupuncture methods (ex. Combinations of acupuncture acupoints, acupuncture methods, different depth of acupuncture needles, treatment according to symptom differentiation) in the field of neuroimaging. There may be specific differences in brain patterns according to acupuncture methods (ex. specificity for acupoints), and such differences may cause distinctive effects in clinical practice. Second, it is important to expand the number of subjects and recruit homogenous subjects. In several studies, only drug-naïve depressed patients were recruited, but most studies contain mixed patients. Also, the number of RCT studies with sufficient subjects in both patients and normal groups is still insufficient. Finally, although several studies investigated long-term ( 8 weeks) effects of acupuncture, it is necessary to identify long-term effects, neuroimaging patterns and elucidate mechanisms of acupuncture through a longer study design of more than 8 weeks.

\section{Conclusion}

This study analyzed eight papers searched from a database to explore the trends of clinical research on acupuncture for depression using neuroimaging techniques. As a result, fMRI was most commonly utilized as a modality. Baihui
(GV20) was the common acupoint for depression in neuroimaging studies. Neuroimaging studies confirmed the neural substrates associated with depression. In addition, genuine acupuncture improved clinical symptoms significantly compared to sham acupuncture. It is found that acupuncture modulates brain activation in a different way from sham group by regulating the activation of defected areas related to emotion, cognition, sensory, DMN. In the future, it is expected to identify the effect of acupuncture on depression by applying large-scale RCT with more homogeneous subjects, various acupuncture methods and neuroimaging techniques.

\section{References}

1. Brenes GA. (2007). Anxiety, depression, and quality of life in primary care patients. Prim Care Companion J Clin Psychiatry, 9(6), 437-443. https://doi.org/10.4088/pcc.v09n0606

2. Kim GE, Jo MW, Shin YW. (2020). Increased prevalence of depression in South Korea from 2002 to 2013. Sci Rep, 10(1), 16979. https://doi.org/10.1038/s41598-020-74119-4

3. 보건복지부. 2021 년 1 분기 코로나 19 국민 정 신건강 실태조사. 2021. Available from: http://www.mohw.go.kr/react/al/sal0301vw.js p?PAR_MENU_ID $=04 \& M E N U \_I D=0403 \& C$ ONT_SEQ=365582\&page $=1$

4. Korean Academy of Medical Sciences. (2021). Evidence-based Recommendations for depression in Primary Care. Seoul, Korea.

5. Duan DM, Tu Y, Jiao S, Qin W. (2011). The relevance between symptoms and magnetic resonance imaging analysis of the hippocampus 
of depressed patients given electro-acupuncture combined with Fluoxetine intervention - A randomized, controlled trial. Chin J Integr Med, 17(3), 190-199. https://doi.org/10.1007/ s11655-011-0666-6

6. Quah-Smith I, Wen W, Chen X, Williams MA, Sachdev PS. (2012). The Brain Effects of Laser Acupuncture in Depressed Individuals: An fMRI Investigation. Med Acupunct. 24(3), 161-171. https://doi.org/10.1089/acu. 2011.0870

7. Quah-Smith I, Suo C, Williams MA, Sachdev PS. (2013). The Antidepressant Effect of Laser Acupuncture: A Comparison of the Resting Brain's Default Mode Network in Healthy and Depressed Subjects During Functional Magnetic Resonance Imaging. Med Acupunct, 25(2), 124-133. https://doi.org/ 10.1089/acu.2012.0901

8. Deng D, Liao H, Duan G, Liu Y, He Q, Liu $\mathrm{H}$, et al. (2016). Modulation of the Default Mode Network in First-Episode, Drug-Naïve Major Depressive Disorder via Acupuncture at Baihui (GV20) Acupoint. Front Hum Neurosci, 10, 230. https://doi.org/10.3389/ fnhum. 2016.00230

9. Wang X, Wang Z, Liu J, Chen J, Liu X, Nie G, et al. (2016). Repeated acupuncture treatments modulate amygdala resting state functional connectivity of depressive patients. Neuroimage Clin, 12, 746-752. https://doi.org/ 10.1016/j.nicl.2016.07.011

10. Wang Z, Wang X, Liu J, Chen J, Liu X, Nie $\mathrm{G}$, et al. (2017). Acupuncture treatment modulates the corticostriatal reward circuitry in major depressive disorder. J Psychiatr Res,
84, 18-26. https://doi.org/10.1016/j.jpsychires. 2016.09.014

11. Duan G, He Q, Pang Y, Chen W, Liao H, Liu H, et al. (2020). Altered amygdala resting-state functional connectivity following acupuncture stimulation at BaiHui (GV20) in first-episode drug-Naïve major depressive disorder. Brain Imaging Behav, 14(6), 2269-2280. https://doi.org/10.1007/s11682-019 $-00178-5$

12. Wei XY, Chen H, Guo C, Tan WL, Zhan SH. (2021). The Instant and Sustained Effect of Electroacupuncture in Postgraduate Students with Depression: An fMRI Study. Neuropsychiatr Dis Treat, 17, 873-883. https://doi.org/10. 2147/NDT.S307083.

13. Emily H, Shunta T, Yuka N \& Christopher P. Tackling the mental health impact of the COVID-19 crisis: An integrated, whole-of-society response. OECD Policy Responses to COVID-19. 2021. Available from: https://www.oecd.org/ coronavirus/policy-responses/tackling-the-men tal-health-impact-of-the-covid-19-crisis-an-int egrated-whole-of-society-response-0ccafa0b/

14. Sheng J, Liu S, Wang Y, Cui R, Zhang X. (2017). The Link between Depression and Chronic Pain: Neural Mechanisms in the Brain. Neural Plast. 2017, 9724371. https://doi.org/10.1155/2017/9724371

15. $\mathrm{EBM}$ 기반 우울증 한의임상진료지침 개발 위원회. (2015). Depression: Korean Medicine Clinical Practice Guideline. Daejeon, Korea. Elsevier Korea.

16. Johnson KA, Fox NC, Sperling RA, Klunk WE. (2012). Brain imaging in Alzheimer disease. Cold Spring Harb Perspect Med, 
2(4), a006213. https://doi.org/10.1101/cshperspect. a006213

17. Anderson KM, Collins MA, Kong R, Fang $\mathrm{K}$, Li J, He T, et al. (2020). Convergent molecular, cellular, and cortical neuroimaging signatures of major depressive disorder. Proc Natl Acad Sci U S A., 117(40), 2513825149. https://doi.org/10.1073/pnas.2008004117

18. Zhuo C, Li G, Lin X, Jiang D, Xu Y, Tian $\mathrm{H}$, et al. (2021). Strategies to solve the reverse inference fallacy in future MRI studies of schizophrenia: a review. Brain Imaging Behav, 15(2), 1115-1133. https://doi.org/10.1007/ s11682-020-00284-9

19. Saeed U, Lang AE, Masellis M. (2020). Neuroimaging Advances in Parkinson's Disease and Atypical Parkinsonian Syndromes. Front Neurol, 11, 572976. https://doi.org/10. 3389/fneur.2020.572976

20. Sun H, Zhao H, Ma C, Bao F, Zhang J,
Wang DH, et al. (2013). Effects of electroacupuncture on depression and the production of glial cell line-derived neurotrophic factor compared with fluoxetine: a randomized controlled pilot study. J Altern Complement Med, 19(9), 733-739. https://doi.org/ 10.1089/acm.2011.0637

21. Lyons Z, van der Watt G, Shen Z, Janca A. (2012). Acupuncture and Chinese herbs as treatments for depression: an Australian pilot study. Complement Ther Clin Pract, 18(4), 216-220. https://doi.org/10.1016/j.ctcp.2012. 06.003

\section{ORCID}

Dong Hyuk Lee https://orcid.org/0000-0003-1602-3138 